\title{
Optical fiber based dosimeter sensor: Beyond TLD-100 limits
}

\author{
G. Amouzad Mahdiraji ${ }^{\mathrm{a}, *}$, M. Ghomeishi $^{\mathrm{a}}$, E. Dermosesian ${ }^{\mathrm{a}}$, S. Hashim ${ }^{\mathrm{b}}$, N.M. Ung ${ }^{\mathrm{c}}$, \\ F.R. Mahamd Adikan ${ }^{\mathrm{a}}$, D.A. Bradley $\mathrm{d}, \mathrm{e}$ \\ a Integrated Lightwave Research Group, Department of Electrical Engineering, Faculty of Engineering, University of Malaya, 50603 Kuala Lumpur, Malaysia \\ b Department of Physics, Universiti Teknologi Malaysia, 81310 Skudai, Johor DarulTakzim, Malaysia \\ ' Clinical Oncology Unit, Faculty of Medicine, University of Malaya, 50603 Kuala Lumpur, Malaysia \\ d Department of Physics, University of Surrey, Guildford GU2 7XH, UK \\ e Department of Physics, Faculty of Science, University of Malaya, 50603 Kuala Lumpur, Malaysia
}

\section{A R T I C L E I N F O}

Article history:

Received 11 June 2014

Received in revised form

22 November 2014

Accepted 26 November 2014

Available online 5 December 2014

\section{Keywords:}

Single mode fiber

Optical fiber application

Dosimeter

Thermoluminescence
A B S T R A C T

This work investigates the suitability of single mode optical fibers (SMFs) as ionizing radiation dosimeter sensors. Thermoluminescence (TL) response studies have been carried out to investigate the performance of two commercial optical fibers, SMF-1 and SMF-2, with different Ge-doping concentrations of 4.9 and $4.3 \mathrm{wt} \%$, respectively, exposed to 0.5 to $8 \mathrm{~Gy}$ doses under 6,9 , and $20 \mathrm{MeV}$ electron radiations. The performance parameters include dose response linearity and sensitivity, energy dependency, glow curve analysis, minimum detectable dose, repeatability, fading effects and optical absorption. The TL dose response of SMF-1, the fiber with the greater Ge concentration of the two, was found to be in excess of 6.3 and 3.2 times that of SMF-2 and TLD-100, respectively. SMF-1 demonstrated capability for detecting a minimum dose of as low as $6 \mathrm{mGy}$, being some 3.2 times superior to that of TLD-100. The results underline the potential of these optical fibers as next-generation alternative dosimeter sensors for detection of ionizing radiation.

(c) 2014 Elsevier B.V. All rights reserved.

\section{Introduction}

Optical fibers have been shown to offer considerable potential as thermoluminescence dosimeters (TLD), with valuable performance and cost advantage over that of well-established commercial TLD materials such as TLD-100. The advantages include high sensitivity to dose, excellent linearity of response over a wide range of dose and low dependence on dose rate [1-3]. A number of groups have investigated the TL and optically stimulated luminescence performance of silica $\left(\mathrm{SiO}_{2}\right)$ optical fibers as radiation dosimeters fo patients undergoing radiotherapy $[4,5]$. Other research groups have worked on these and nuclear track detectors for fission fragments for in situ measurements of nuclear reactors [6,7]. In addition, others have reported on the designing of special photo-, radioand/or thermo-luminescence material with high sensitivity in measuring irradiation dose, including phosphate glasses doped with lithium and barium [8], zirconium oxide $\left(\mathrm{ZrO}_{2}\right)$ [9], copper activated calcium borate $\left(\mathrm{CaB}_{4} \mathrm{O}_{7}: \mathrm{Cu}\right)$ nanocrystals [10], manganese doped calcium tetraborate $\left(\mathrm{CaB}_{4} \mathrm{O}_{7}: \mathrm{Mn}\right)$ nonocrystal [11], lithium

\footnotetext{
* Corresponding author. Tel.: +60 126274711.

E-mail addresses: ghafouram@gmail.com, ghafour@um.edu.my (G.A. Mahdiraji).
}

potassium borate glass doped with titanium oxide $\left(\mathrm{TiO}_{2}\right)$ and magnesium oxide (MgO) [12], double potassium yttrium fluoride $\left(\mathrm{K}_{2} \mathrm{YF}_{5}\right)$ crystals doped with samarium $\left(\mathrm{Sm}^{3+}\right)$ and terbium $\left(\mathrm{Tb}^{3+}\right)$ ions [13], all emphasizing the importance of producing a dosimeter with high sensitivity.

Our current work focuses on the investigation of two types of standard single mode fibers, SMF-1 and SMF-2, which have been subjected to 6,9 and $20 \mathrm{MeV}$ electron irradiations at doses up to $8 \mathrm{~Gy}$, comparison being made with the performance of TLD-100. The intention is to emphasise that these relatively cheap commercially available telecommunication optical fibers can be used as alternatives to the more conventional phosphor-based TL materials such as TLD-100 for dosimetric applications.

\section{Materials and methods}

\subsection{Elemental analysis}

Two types of commercially available Ge-doped standard SMFs namely SMF-1 and SMF-2 are used in this study. Both SMFs have similar core and cladding diameters of about $8.5 \mu \mathrm{m}$ and $125 \mu \mathrm{m}$, respectively. Energy dispersive X-ray (EDX) analysis was used for elemental measurement. For each SMF type, five sets of EDX 
Table 1

EDX analysis of SMF-1 and -2 .

\begin{tabular}{|c|c|c|c|c|c|c|c|c|c|c|c|c|}
\hline & \multicolumn{6}{|c|}{ SMF-1 } & \multicolumn{6}{|c|}{ SMF-2 } \\
\hline & \multicolumn{3}{|c|}{ Weight (\%) } & \multicolumn{3}{|c|}{ Atomic (\%) } & \multicolumn{3}{|c|}{ Weight (\%) } & \multicolumn{3}{|c|}{ Atomic (\%) } \\
\hline & 0 & $\mathrm{Si}$ & $\mathrm{Ge}$ & 0 & $\mathrm{Si}$ & Ge & 0 & $\mathrm{Si}$ & Ge & 0 & $\mathrm{Si}$ & Ge \\
\hline Sample 1 & 69.6 & 25.5 & 4.9 & 81.7 & 17.0 & 1.3 & 70.2 & 25.2 & 4.7 & 82.0 & 16.8 & 1.2 \\
\hline Sample 2 & 69.6 & 25.5 & 4.8 & 81.7 & 17.1 & 1.3 & 66.0 & 30.1 & 3.9 & 78.6 & 20.4 & 1.0 \\
\hline Sample 3 & 72.0 & 22.7 & 5.4 & 83.6 & 15.0 & 1.4 & 66.2 & 28.9 & 5.0 & 79.1 & 19.6 & 1.3 \\
\hline Sample 4 & 52.8 & 42.7 & 4.6 & 67.6 & 31.2 & 1.3 & 50.6 & 45.5 & 3.9 & 79.1 & 19.6 & 1.3 \\
\hline Sample 5 & 53.1 & 42.3 & 4.8 & 67.7 & 30.9 & 1.4 & 51.8 & 44.1 & 4.1 & 65.4 & 33.5 & 1.1 \\
\hline Average & 63.4 & 31.7 & 4.9 & 76.5 & 22.2 & 1.3 & 60.9 & 34.7 & 4.3 & 76.8 & 22.0 & 1.2 \\
\hline STD & 9.6 & 9.9 & 0.3 & 8.1 & 8.1 & 0.1 & 9.1 & 9.4 & 0.5 & 6.5 & 6.6 & 0.1 \\
\hline
\end{tabular}
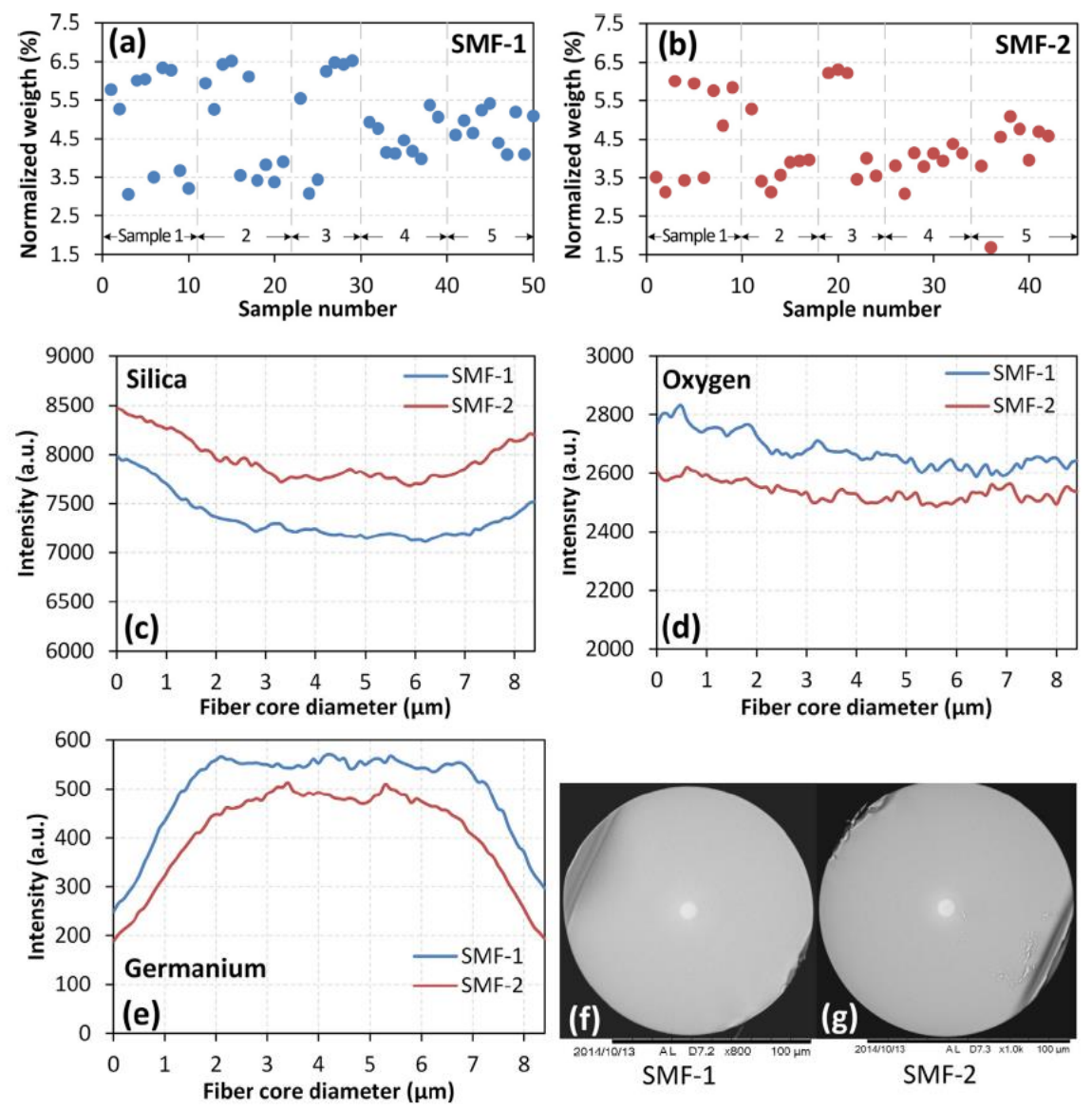

Fig. 1. Elemental concentration distribution across the fiber core cross section, obtained using EDX. (a) and (b) Normalized weight (\%) Ge concentration for 50 and 42 ROIs measured within fiber core area of five different fiber samples for SMF-1 and -2, respectively. The result of Rols per fiber sample are identified in (a) and (b). (c), (d), and (e) showing silica, oxygen, and germanium intensity concentration, respectively, measured by line scanning across fiber core area (averaged from fiber Samples 4 and 5 in Table 1). (f) and ( $\mathrm{g}$ ) Show cross section area of SMF-1 and -2 taken by SEM imaging.

measurement were done using different samples of the same fiber type. For each fiber sample, 6-10 region-of-interests (ROIs) are selected within the fiber core area. Table 1 shows the results of average compositional analysis over all ROIs per fiber sample for SMF-1 and -2. On average, SMF-1 and -2 indicate the presence of $\mathrm{Ge}$ at about 4.9 and $4.3 \mathrm{wt} \%$, respectively. Details of the Ge concentration variability per ROI for the five samples are presented in Fig. 1(a) and (b) for SMF-1 and -2, respectively. Over and above the overall average value presented in Table 1, it is noted that the majority of Ge concentrations in SMF-1 are found to be greater than that in SMF-2. As an instance, 22 out of the 50 readings made in SMF-1 showed a concentration greater than $5 \mathrm{wt} \%$ compared to 9 out of 42 readings in SMF-2. In regard to concentrations of $\sim 4 \mathrm{wt} \%$, there are 19 over 42 readings for SMF- 2 and 12 over 50 readings 
for SMF-1. This suggests that the concentration of Ge in SMF- 1 and -2 can best be characterised as $\sim 5$ and $4 \mathrm{wt} \%$, respectively. Fig. 1(c) to (e) illustrate the result of $\mathrm{Si}, \mathrm{O}$, and $\mathrm{Ge}$ concentrations across the fiber core surface, respectively, obtained as averages of line scanning over the cross-sections of fiber Samples 4 and 5 under EDX inspection. Results in Table 1 and Fig. 1 are in agreement that $\mathrm{Ge}$ and 0 concentrations in the SMF- 1 core area are greater than that in the SMF-2 core area, while Si is greater in SMF-2. It should be noted that the EDX analysis over fiber cladding is also performed. However, almost similar $\mathrm{Si}$ and $\mathrm{O}$ concentrations are observed for both fiber samples in the absence of detecting any other elements. Fig. 1(f) and (g) shows SMF-1 and -2's cross sections obtained by SEM imaging.

\subsection{Absorption}

Since the luminescence generated from an optical fiber dosimeter is contributed by the structural defects in the fiber, having fibe spectral absorption would be useful in analyzing the type of defect centers associated in each optical fiber samples. Fig. 2 illustrates the extent of light absorption detected in $8 \mathrm{~m}$ of SMF-1 and -2 normalized per unit length. The absorption presented here is obtained by measuring optical fiber attenuation and subtracting the attenuation from the reference light source. The fiber attenuation was measured by directly connecting one end of each fiber to the pigtail of a supercontinuum light source (NKT Photonics) and the other end to an Optical Spectrum Analyzer (OSA). The attenuation spectrum of each fiber is then subtracted from the emitted light source /reference spectrum, which is measured by directly connecting the supercontinuum source pigtail to OSA. The spectrum for SMF- 1 shows much greater absorption compared to SMF-2, which implies the presence of more defect centers in SMF-1. Considering the spectral range of TLD reader used in this study (i.e., around $270-630 \mathrm{~nm}$ ); the main bands in SMF-1 with elevated absorption are around $560-630 \mathrm{~nm}$, $500 \mathrm{~nm}, 485 \mathrm{~nm}, 455 \mathrm{~nm}$. In general, the defect centers associated with these absorption bands can be referred to as non-bridging oxygen hole center ( $\mathrm{NBOHC}$ ) with absorption bands within around $550-690 \mathrm{~nm}$ and Ge-related defect centers around $570-410 \mathrm{~nm}$ [14]. The fiber absorption spectra show greater absorption band at the longer wavelengths, however, the TL generated from these absorption bands is not detectable with the TLD reader used in this study.

\subsection{Sample preparation}

Prior to irradiation, the optical fiber samples were prepared, first by carefully removing the outer polymer coating to the optical fiber. This is done by using a fiber stripper that provides for removal of the buffer coating, without scratching or nicking of the glass fiber. A cotton cloth containing methyl alcohol was then used to clean the stripped fibers in order to ensure complete removal of any residua polymer or impurities. The fibers were then manually cut using a diamond cone-point cutter, to the length of $(5 \pm 0.5) \mathrm{mm}$ as dictated by the maximum size of the square planchet of TL reader.

All fiber samples were then annealed before the irradiation stage in order to standardize their thermal history. This allows for removal of any residual TL signal from previous handling (the socalled mechanical/triboluminescence signal) and elimination of the unstable low-temperature glow curve component [15]. The annealing process for fibers is carried out using a furnace operating with a time-temperature profile of $400^{\circ} \mathrm{C}$ for $1 \mathrm{~h}$, subsequently left to cool down to room temperature. The TLD-100 chips were annealed for $1 \mathrm{~h}$ at $400^{\circ} \mathrm{C}$ and subsequently for $2 \mathrm{~h}$ at $100^{\circ} \mathrm{C}$. After cooling, 7-10 pieces of the samples were then placed in small plastic bags, ready for irradiation and subsequent evaluation of the mean TL yield. When not being handled, all samples were placed in a light-tight container to prevent unnecessary exposure to light as this could influence the TL results.

\subsection{Irradiation}

The SMF-1, SMF-2 and TLD-100 samples were placed at the surface of a solid water ${ }^{T M}$ phantom whose function is to provide for the standardized full-scatter condition (reference conditions) as conventionally adopted and were exposed to 6,9 and $20 \mathrm{MeV}$ electron beams, $600 \mathrm{cGy} / \mathrm{min}$ dose rate, with accumulation doses from 0.5 to $8 \mathrm{~Gy}$, delivered by a Varian Model $2100 \mathrm{C}$ linear accelerator (Varian Medical System, Palo Alto, USA) located at the University of Malaya Medical Centre. One monitor unit corresponds to dose of $1 \mathrm{cGy}$ delivered under the reference conditions. Bolus thicknesses of $1.5,1.5$ and $2.5 \mathrm{~cm}$ were used as build-up medium during irradiation using the 6,9 and $20 \mathrm{MeV}$ beams, respectively. A field size of $20 \times 20 \mathrm{~cm}^{2}$, source to skin distance of $100 \mathrm{~cm}$ and applicator size of $20 \times 20 \mathrm{~cm}^{2}$ were used for all irradiations.

It should be noted that the recorded absolute radiation dose delivered by the linac is based on adoption of the procedures detailed in the International Atomic Energy Agency Report TRS398, the output being ensured to be within $\pm 2 \%$ of the intended delivered dose. Measurements of dose from electron beam irradiation have been performed by the in-house medical physicists, supported by monthly quality assurance $(\mathrm{QA})$ checks made using a Roos ionization chamber IBA PPC40 with a Supermax electrometer.

\subsection{TL measurements}

After the exposures and following a selected delay of $24 \mathrm{~h}$ (to allow uniform control of thermal fading), the optical fiber TL yield was read out using a Harshaw $3500 \mathrm{TL}$ reader. In this study, the time temperature profile (TTP) was set as follows: preheat temperature of $50^{\circ} \mathrm{C}$, maximum temperature of $400^{\circ} \mathrm{C}$, acquired temperature rate at $25^{\circ} \mathrm{C} / \mathrm{s}$, post annealing of $6 \mathrm{~s}$ and total acquisition time of $20 \mathrm{~s}$. The readings were performed under nitrogen gas flow to suppress possible spurious light signals from triboluminescence and also to reduce oxidation of the heating element.

All TL responses were then normalized to the mass of the sample. An accurate electronic balance with $0.1 \mathrm{mg}$ accuracy was used to measure the mass of a group of 10-15 randomly selected fiber samples per fiber type. For simplicity in this study, mean masses of $0.132 \mathrm{mg}$ for SMF-1 and $0.136 \mathrm{mg}$ for SMF-2 were used to normalize the TL yield for individual fiber samples. However, for TLD-100 chips, since the weight per chip is significantly high ( $\sim 23-24 \mathrm{mg}$ ), the normalization is performed individually using actual weight per chip.

\section{Results}

\subsection{Dose response}

Fig. 3 shows TL response of the two SMFs for the three different electron energy irradiations, 6,9 and $20 \mathrm{MeV}$, in comparison with TLD-100 TL yields. In terms of linearity, all samples show linear response (linear fitting curve $R^{2}>97.8 \%$ ) over the investigated dose range, 0.5 to $8 \mathrm{~Gy}$ for all three energies. Taking TLD-100 as the benchmark, SMF-1 exhibits significantly greater response to the TLD-100, while SMF-2 has approximately half of the response of the TLD-100. On average, SMF-1 has a TL response that is about 3.2 times greater than that for TLD-100 at 6,9 and $20 \mathrm{MeV}$, and the results also confirm SMF-1 to significantly outperform TLD100. The potential of Ge-doped optical fiber as TLD has been reported earlier in the literature. Hashim et al. $[15,16]$ compared the TL response of a Ge-doped commercial SMF with TLD-100, oxygen- and Al-doped fibers under electron and photon energies. 


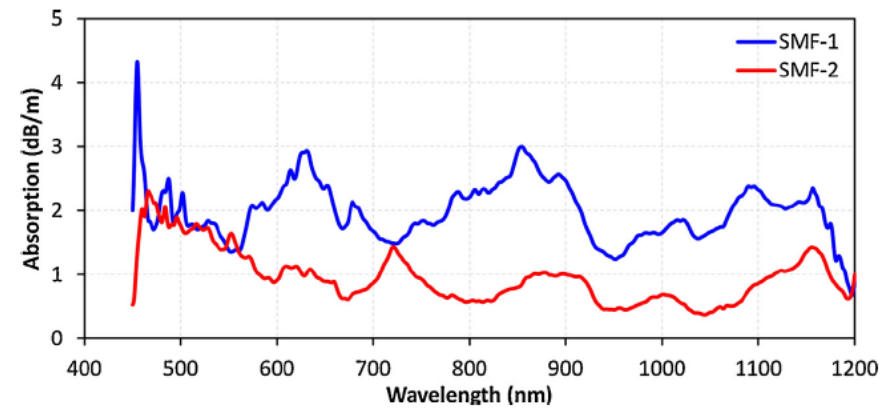

Fig. 2. Absorption spectrum of SMF-1 and -2.

Ge-doped fiber showed significantly better TL response compared to oxygen- and Al-doped fibers but showed a TL yield about 8 times lower compared to TLD-100. In another study, Yaakob et al. [17] confirmed the significant outperformance of Ge-doped fibers compared to Al-doped fiber, while the Ge-doped fiber used in their study could yield a TL half that of a TLD- 100 chip. To the best of our knowledge there exist no previous reports showing SMFs to outperform TLD-100, certainly not to the present extent. Recently, Benabdesselam et al. [1] reported TL glow curve analysis of a multimode fiber (MMF) with $62.5 \mu \mathrm{m}$ diameter with 2-layer Gedoped fiber compared with TLD-500 and -600 . The MMF is shown to be relatively more sensitive compared to TLD-500 and -600. Zahaimi et al. [18] demonstrated that the TL yield in a SMF with $8-9 \mu \mathrm{m}$ diameter can be improved upon by up to 6 times using a larger core MMF with $50 \mu \mathrm{m}$ diameter with the same cladding size.
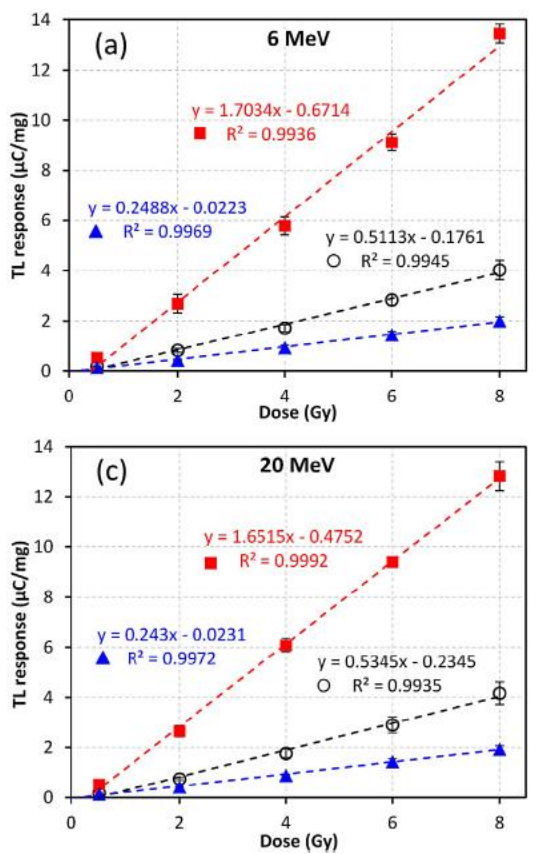

Fig. 4 (left side) show the energy dependency of the two SMFs compared to that of TLD-100 at 6, 9 and $20 \mathrm{MeV}$. Both SMFs show low sensitivity to change in radiation energy, sharing this dosimetrically favorable behavior with that of TLD-100. On the other hand, Fig. 4 (right side) show the sensitivity curve of the optical fibers and TLD-100 calculated from their TL response divided by relative applied dose. Besides the irradiation energy insensitivity, both SMF-1 and TLD-100 have a positive sensitivity slope compared to SMF-2. Unlike SMF-2, SMF-1 and TLD-100 have slightly higher sensitivity at the higher doses compared to lower dose, showing them to be slightly dose dependent.

Uncertainty of the slope $(\Delta S)$ of the fitted curve in TL response (shown in Figs. 3 and 4 (left)) and dose detection sensitivity (shown in Fig. 4 (right)) is calculated based on the maximum slope $\left(S_{\max }\right)$ and minimum slope $\left(S_{\min }\right)$ calculated based on the variation or standard deviation (STD) in TL response as

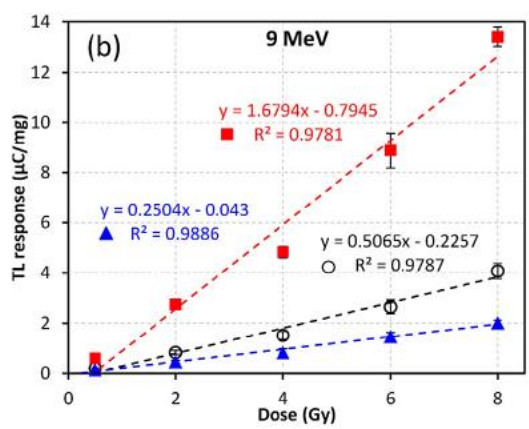

$$
\begin{aligned}
& \text { ○ TLD-100 } \\
& \text { - SMF-1 } \\
& \text { - SMF-2 } \\
& \text { - - - Linear (TLD-100) } \\
& \text { - - - - Linear (SMF-1) } \\
& \text {-...- Linear (SMF-2) }
\end{aligned}
$$

Fig. 3. TL response of two different SMFs in comparison with TLD-100 irradiated to 6, 9 and $20 \mathrm{MeV}$ electrons. SMF-1 shows significantly greater TL response than SMF-2 and TLD-100. 

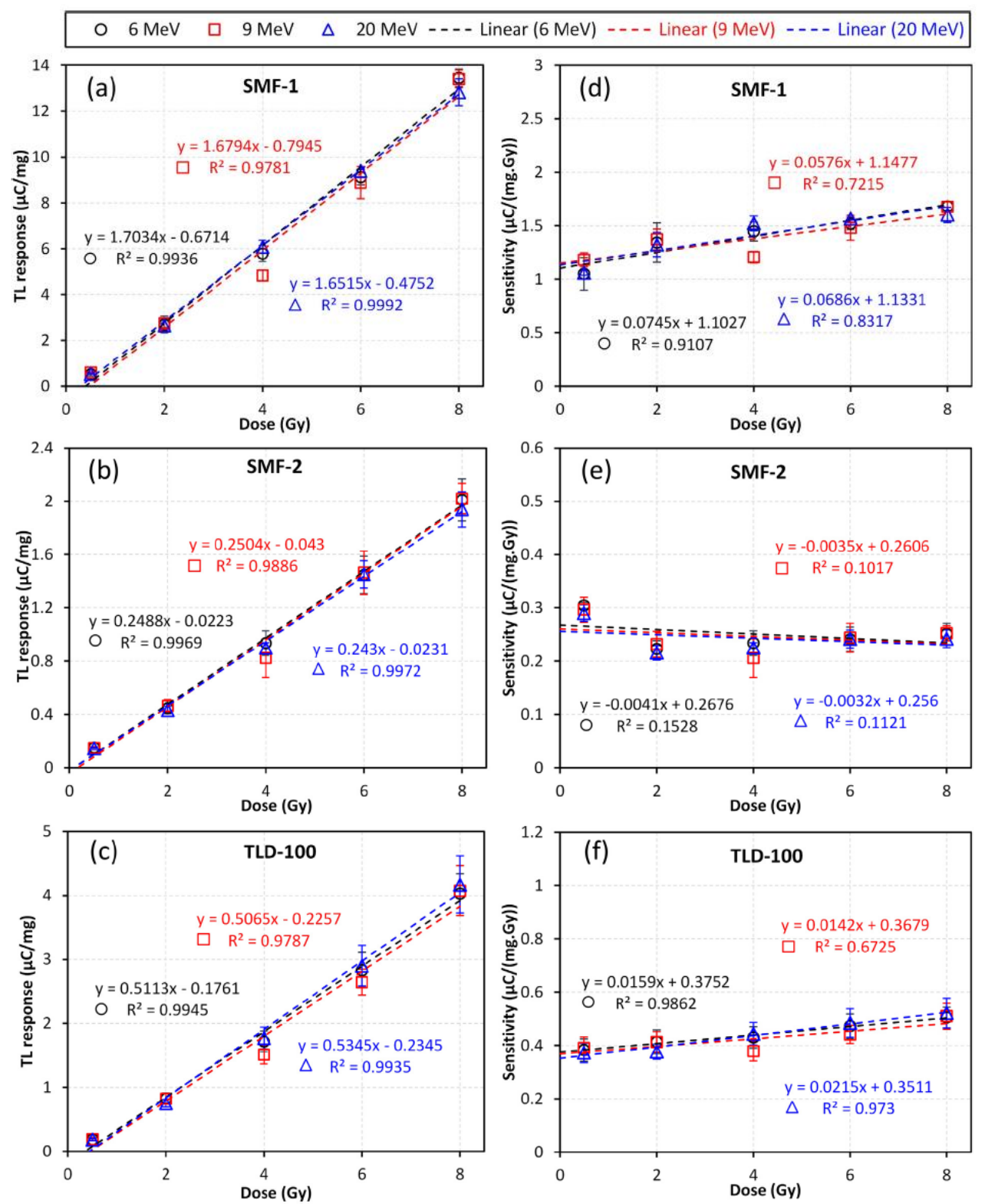

Fig. 4. Comparison of energy dependencies of SMF-1, SMF-2, and TLD-100 for electron irradiations between 6 and $20 \mathrm{MeV}$.

$\Delta S=\left(S_{\max }-S_{\min }\right) / 2$, where $S_{\max }=\left(y_{\max a t x_{\min }} / y_{\min a t} x_{\min }\right)$ and $S_{\min }=\left(y_{\min a t} x_{\max } / y_{\max a t} x_{\min }\right)$. The $y_{\max a t} x_{\max }$ is the maximum value in $y$-axis (mean +STD) at maximum value in $x$-axis, which here refers to the maximum TL value at dose $8 \mathrm{~Gy}$ and the $y_{\min }$ at $x_{\min }$ is the minimum value in $y$-axis (mean - STD) at minimum value in $x$-axis, which here refers to the minimum TL value at dose $0.5 \mathrm{~Gy}$.

Table 2 shows the slope of fit and its uncertainty for TL response (Table 2(a)) and dose sensitivity (Table 2(b)) for SMF-1, -2, and
TLD-100 for the three radiation energies. The highest variation in the slope of the fit to the TL responses for SMF-1, -2 and TLD-100 are $6 \%, 2 \%$ and $5 \%$; and to the dose sensitivity are $2 \%, 0.4 \%$ and $1 \%$, respectively. These low variations in the slope of the fit reconfirm the stability of the SMFs in terms of both energy independency and dose sensitivity, comparable with commercially available TLD-100.

Table 3 shows the average sensitivity of SMFs compared to TLD100 , obtained by dividing the TL response by its corresponding dose 
Table 2

The slope and its uncertainty for (a) the fitted curves in TL response and (b) the fitted curves in sensitivity for SMF- $1,-2$, and TLD-100 for the three electron radiation energies.

\begin{tabular}{lccc}
\hline (a) & $\begin{array}{l}\text { TL response } \\
6 \mathrm{MeV}\end{array}$ & $9 \mathrm{MeV}$ & $20 \mathrm{MeV}$ \\
\hline SMF-1 & $1.70 \pm 0.05$ & $1.68 \pm 0.04$ & $1.65 \pm 0.06$ \\
SMF-2 & $0.25 \pm 0.02$ & $0.25 \pm 0.01$ & $0.24 \pm 0.02$ \\
TLD-100 & $0.51 \pm 0.04$ & $0.51 \pm 0.04$ & $0.53 \pm 0.05$ \\
(b) & & & \\
& Sensitivity & & \\
SMF-1 & $0.075 \pm 0.020$ & $0.058 \pm 0.012$ & $0.069 \pm 0.010$ \\
SMF-2 & $0.004 \pm 0.003$ & $0.004 \pm 0.004$ & $0.003 \pm 0.003$ \\
TLD-100 & $0.016 \pm 0.008$ & $0.014 \pm 0.009$ & $0.022 \pm 0.010$ \\
\hline
\end{tabular}

Table 3

The average sensitivity of SMF-1, -2 , and TLD-100 with STD.

\begin{tabular}{llll}
\hline & SMF-1 & TLD100 & SMF-2 \\
\hline Sensitivity $(\mu \mathrm{C} /(\mathrm{mg}$ Gy) $)$ & 1.40 & 0.44 & 0.25 \\
STD & 0.08 & 0.04 & 0.02 \\
\hline
\end{tabular}

over the three radiation energies. SMF-1 offers a dose sensitivity 3.2 and 5.6 times that of TLD-100 and SMF-2, respectively, and TLD100 shows about 1.7 times more sensitivity compared to SMF-2. The response variation in the dosimeter samples with greater sensitivity is greater than that of the dosimeters with lower sensitivity.

Since the fiber samples were cut manually to a mean length of $5 \mathrm{~mm}$ with a tolerance of $0.5 \mathrm{~mm}$, for both SMFs the non-uniformity in length results in a greater variation in TL response compared to TLD-100. Due to very low mass of individual fibers, in present practice the mean mass has been used to normalize each fiber sample, instead of seeking to obtain individual fiber corrections. It should be noted that although, the fiber samples are cut manually, the highest measured TL variation observed was less than $8 \%$, which is related to $6 \mathrm{~Gy}, 9 \mathrm{MeV}$, SMF-1 (Fig. 3(b)) with STD of 0.7 and TL yield of $8.9 \mu \mathrm{C} / \mathrm{mg}$. This variation in TL yield of optical fiber can be reduced by cutting the fiber samples with an automated fiber cleaver.

\subsection{Glow curve analysis}

Fig. 5 shows the glow curves of SMF-1, -2, and TLD-100 resulting from electron irradiation at $6 \mathrm{MeV}$, delivering doses of 0.5 , $2,4,6$, and $8 \mathrm{~Gy}$. To provide intercomparison between all three samples, the curves have been normalized to dosimeter mass, the time-temperature profile covering temperatures from $50^{\circ} \mathrm{C}$ to $400^{\circ} \mathrm{C}$. Compared with TLD-100, with thermal luminescence at well-defined temperatures, at around $170,220,260$ and $330^{\circ} \mathrm{C}$, it is apparent that the SMFs exhibit a broad range of thermal excitation, as expected of an amorphous system.

Using the so-called computerized glow curve deconvolution (CGCD) method, second derivative deconvolution of the glow curve of SMF-1 and -2 has been carried out, Fig. 6, respectively, showing the resultant component peaks. The trap parameters associated with the component peaks are shown in Table 4, a mixture of first and second order kinetics being applied. It is found that the first half of the glow curve follows first order kinetics since while the second half follows second order kinetics. The deviation of the fitted sub-component peaks from the gross glow curve is about $0.04 \%$, with a figure of merit of $2.8 \%$.

In SMF-1, glow curve peak number 1, with activation energy $1.6 \mathrm{eV}$, could be associated with $\mathrm{Si}$ nanoclusters, while peak number 2 , activation energy $1.8 \mathrm{eV}$, could also be related to Si nanoclusters and the oxygen-deficiency center (ODC) in the silica. Peaks numbers 3 and 4, activation energy 2.6 and $2.4 \mathrm{eV}$ respectively, are
Table 4

Glow peak analysis of SMF-1 and SMF-2. $b$ Is the kinetics order of the glow peaks, $E$ is the activation energy of each trap, in units of $(\mathrm{eV}), s$ is the frequency factor $\left(\mathrm{s}^{-1}\right)$, $n_{0}$ is the initial concentration of trapped electrons $\left(\mathrm{cm}^{-3}\right)$, Peak-I is the maximum intensity of glow peak, in units of (nC), Peak- $T$ is the temperature at maximum intensity of the glow peak in $\left({ }^{\circ} \mathrm{C}\right)$, FWHM is the full width at half maximum intensity of the glow peak, also in units of $\left({ }^{\circ} \mathrm{C}\right)$, and the peak emission wavelength in $\mathrm{nm}$.

\begin{tabular}{lllllll}
\hline SMF-1 & Trap: $\mathbf{1}$ & Trap: $\mathbf{2}$ & Trap: $\mathbf{3}$ & Trap: $\mathbf{4}$ & Trap: $\mathbf{5}$ & \\
\hline $\boldsymbol{b}$ & 1st Order & 1st Order & 2nd Order & 2nd Order & 2nd Order & \\
$\boldsymbol{E}$ & 1.6 & 1.8 & 2.6 & 2.4 & 2.2 & \\
$\boldsymbol{s}$ & $1.3 \mathrm{E}+6$ & $3.5 \mathrm{E}+7$ & $9.5 \mathrm{E}+7$ & $2.2 \mathrm{E}+5$ & $7.7 \mathrm{E}+2$ & \\
$\boldsymbol{n}_{\boldsymbol{0}}$ & $2.9 \mathrm{E}+6$ & $5.5 \mathrm{E}+6$ & $4.4 \mathrm{E}+6$ & $4.7 \mathrm{E}+6$ & $5.1 \mathrm{E}+6$ & \\
Peak-I & 933 & 1874 & 1693 & 1447 & 1195 & \\
Peak- & 177 & 227 & 277 & 307 & 362 & \\
FWHM & 115 & 110 & 100 & 115 & 132 & \\
Emission & 775 & 689 & 477 & 517 & 564 & \\
SMF-2 & Trap: $\mathbf{1}$ & Trap: $\mathbf{2}$ & Trap: $\mathbf{3}$ & Trap: $\mathbf{4}$ & Trap: $\mathbf{5}$ & Trap: $\mathbf{6}$ \\
$\boldsymbol{b}$ & $1 \mathrm{~s}$ Order & 1st Order & 1st Order & 2nd Order & 2nd Order & 2nd Order \\
$\boldsymbol{E}$ & 2.5 & 1.5 & 2.0 & 2.2 & 2.5 & 3.5 \\
$\boldsymbol{s}$ & $6.1 \mathrm{E}+13$ & $1.8 \mathrm{E}+5$ & $1.2 \mathrm{E}+10$ & $6.2 \mathrm{E}+3$ & $8.7 \mathrm{E}+6$ & $2.1 \mathrm{E}+16$ \\
$\boldsymbol{n}_{\boldsymbol{0}}$ & $4.6 \mathrm{E}+5$ & $6.8 \mathrm{E}+5$ & $4.7 \mathrm{E}+5$ & $6.2 \mathrm{E}+5$ & $7.1 \mathrm{E}+5$ & $4.0 \mathrm{E}+5$ \\
Peak-I & 172 & 147 & 275 & 265 & 228 & 150 \\
Peak- $\boldsymbol{T}$ & 392 & 227 & 177 & 307 & 277 & 362 \\
FWHM & 70 & 50 & 42.5 & 40 & 62.5 & 77.5 \\
Emission & 496 & 827 & 620 & 564 & 496 & 354 \\
\hline
\end{tabular}

probably due to the ion dopants $\mathrm{Ge}^{+}$and/or $\mathrm{Si}^{+}$, peak number 3 being also probably related to the self-trapped exciton (STE) defect in silica. Peak number 5, with energy $2.2 \mathrm{eV}$, could be due to the STE, Si implantation, Si nanoclusters, and/or hydrogen defects [19].

In SMF-2, peak numbers 1 and 5 with 1 st and 2nd order activation energy of $2.5 \mathrm{eV}$ could be associated with the STE, Si implantation, and/or Si nanoclusters. Peak number 2, with activation energy $1.5 \mathrm{eV}$, is suggested to be due to $\mathrm{Si}$ nanoclusters in the fiber. Peak number 3 and 4, with activation energy 2.0 and $2.2 \mathrm{eV}$, is suggested to be mainly due to the STE, the ion dopant Si and C implantation, Si nanoclusters and/or hydrogen defects [19].

\subsection{Minimum detectable dose}

Minimum detectable dose (MDD) or the lower dose detection limit of a dosimeter is of clear importance in efforts toward reducing the limit of a dosimetric system for lower dose applications. In addition to the slope of TL response $(m)$ of a dosimeter, the MDD also depends on the TL background signal, calculated as

$\mathrm{MDD}=\frac{1}{m}\left(B_{\text {mean }}+2 \sigma\right)$

where $B_{\text {mean }}$ is the average of the TL background signal combined with the photo-multiplier tube (PMT) noise signal obtained from TL samples annealed but unirradiated or the TL value provided by the TLD reader in the absence of any TL sample within the reader and $\sigma$ is the standard deviation of the background signals. The average and STD of background noise for the TLD reader used in this study are $7.9 \mathrm{nC}$ and 0.83 , respectively. On the other hand, the variation in the slope of the fit in the TL response reflects uncertainty on the MDD. Considering the slope of the fitted curve and its variation presented in Table 2, the MDD and its variation for SMF-1, -2 , and TLD-100 over three applied energies are estimated based on Equation (1)

Table 5

Minimum detectable dose and its tolerance for SMF-1, -2, and TLD-100 for 6, 9, and $20 \mathrm{MeV}$ electron irradiations.

\begin{tabular}{|c|c|c|c|c|}
\hline \multicolumn{5}{|c|}{ Minimum detectable dose (mGy) } \\
\hline & $6 \mathrm{MeV}$ & $9 \mathrm{MeV}$ & $20 \mathrm{MeV}$ & Mean \\
\hline SMF-1 & $6 \pm 0$ & $6 \pm 0$ & $6 \pm 0$ & $6 \pm 0$ \\
\hline SMF-2 & $39 \pm 3$ & $38 \pm 2$ & $39 \pm 3$ & $39 \pm 3$ \\
\hline TLD-100 & $19 \pm 1$ & $19 \pm 2$ & $18 \pm 2$ & $19 \pm 2$ \\
\hline
\end{tabular}

Link to Full-Text Articles :

http://www.sciencedirect.com/science/article/pii/s0924424714004993 